\title{
Modelling and analysis of the effects of aerosols in making artificial rain
}

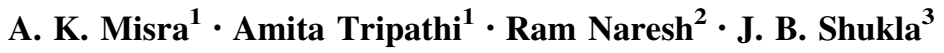

Received: 6 September 2016/Accepted: 27 September 2016/Published online: 7 October 2016

(c) Springer International Publishing Switzerland 2016

\begin{abstract}
To overcome the water crisis for irrigation and other purposes, in this paper, we propose a non-linear mathematical model for artificial rain making by considering five dependent variables namely, water vapor density, densities of cloud droplets of small and large sizes, density of rain drops and cumulative concentration of mixture of aerosols of different sizes. It is assumed that these aerosols are conducive to the process of rain making, i.e. (a) the formation of small size cloud droplets from water vapors through the processes of nucleation and condensation, (b) changing them into large size cloud droplets through the processes of condensation, agglomeration, etc., and (c) changing these large cloud droplets into rain drops. The proposed model is analyzed using stability theory of differential equations. It is found that only one equilibrium is feasible and sufficient conditions for stability of such equilibrium are obtained. It is shown that the intensity of rainfall increases as the cumulative concentration of externally introduced aerosols in the atmosphere increases. Analysis reveals that for the continuous rainfall, it is
\end{abstract}

\author{
A. K. Misra \\ akmisra@bhu.ac.in \\ Amita Tripathi \\ amita4utripathi@gmail.com \\ Ram Naresh \\ ramntripathi@yahoo.com \\ J. B. Shukla \\ jbs@iitk.ac.in \\ 1 Department of Mathematics, Institute of Science, Banaras \\ Hindu University, Varanasi 221 005, India \\ 2 Department of Mathematics, H.B. Technological Institute, \\ Kanpur 208 002, India \\ 3 C-MEND, 18, Navsheel Dham, Kanpur 208 017, India
}

necessary that water vapors must be continuously formed in the atmosphere. The numerical simulation of the model supports the analytical results.

Keywords Mathematical model $\cdot$ Artificial rain $\cdot$ Auto conversion $\cdot$ Aerosols $\cdot$ Stability

\section{Introduction}

Water is essential for life on the planet Earth. Abundant amount of water is available in oceans but it is salty and useless for human population, animal population, agricultural purposes, etc. The water available in lakes, ponds, rivers and inside the ground is used for different purposes by human and animal population including irrigation of agricultural forms. These sources of water are re-charged by rainfall during monsoon rain. The economy of several Asian countries including India depends on monsoon rain. There are some regions in India, e.g. Bundelkhand (Uttar Pradesh), Marathwada, Vidarbh (Maharashtra), etc., where farmers completely depend on monsoon rain for irrigation. In the last few years, much less amount of rainfall is observed in these regions, causing draught like situation and hence very little agricultural production (Maps of India 2016).

Rainfall is a very important but complex phenomenon in nature. Various physicochemical processes, like condensation, nucleation, agglomeration, etc. are involved in the rainfall (Pandis et al. 1990; Pruppacher and Klett 1997). First, tiny cloud droplets are formed from water vapors through the processes of condensation and nucleation. These tiny droplets move randomly within the cloud, collide and stick with each other, which results the formation of large size cloud droplet. Further, these large size cloud 
droplets are converted into raindrops through the processes of nucleation and agglomeration. Aerosols play an important role in all these processes and thus in the rainfall. Aerosols are fine particles of liquid or solid with diameters in the range $10^{-9}-10^{-4} \mathrm{~m}$ and originate from natural and anthropogenic sources and are also formed in the atmosphere due to conversion of gas into particles through chemical reactions. The primary components of aerosol particles are dust, crustal elements, nitrate, sodium chloride and biogenic organic particles such as pollen, spores and plant fragments. These aerosols increase the condensation of water vapors as cloud droplets are formed around aerosols (Finlayson-Pitts and Pitts 2000; Houghton et al. 2001; Lohmann and Feichter 2005; Maps of India 2005).

In some places of the world, experiments have been performed for making artificial rain using different kinds of aerosols, like calcium chloride and silver iodide, etc. (Xinhua News Agency 2006). Generally, three stages are involved in the process of making artificial rain. In the first stage, water vapors condense in presence of artificially introduced aerosols of calcium chloride, calcium oxide, a compound of salt and urea to form small size cloud droplets. In the second stage, large size cloud droplets are formed from small size cloud droplets using the aerosols such as dry ice, kitchen salt or even calcium chloride. In the third stage, large size cloud droplets are converted into raindrops using the aerosols, like silver iodide and dry ice (Narasimhan 2002; Xinhua News Agency 2006). These aerosols may be introduced in the local atmosphere either by using small aircrafts or by firing sticks filled with the aerosols from the ground.

Some theoretical as well as laboratory experiments have been conducted to study the role of aerosols in cloud condensation nuclei (CCN) under different atmospheric conditions (Easter and Hobbs 1974; Flossmann et al. 1987; Shaw 1989; Pandis et al. 1990; Hegg 1990, 2001; Hudson and Frisbie 1991; Lelieveld and Heintzenberg 1992; Saxena and Grovenstein 1994; Coffman and Hegg 1995; Pruppacher and Klett 1997; Bower et al. 1997). In all these studies, it is found that aerosols act as Cloud Condensation Nuclei (CCNs), around which cloud droplets are formed. In particular, Hegg $(1990 ; 2001)$ has studied the production of cloud condensation nuclei in the marine atmosphere and the impact of clouds on aerosols. Similarly, Shaw (1989) has investigated the production of condensation nuclei in clean air by nucleation of sulphuric acid. Further, it may be noted that some modeling studies have been conducted for the removal of pollutants from the atmosphere (Shukla et al. 2008; Sundar et al. 2009; Agarwal and Tandon 2010) and references therein using wind and rain. Agarwal and Tandon (2010) have studied the dispersal of air pollutants in the atmosphere due to the wind. Sundar et al. (2009) have studied the removal of gaseous pollutants from the atmosphere by considering their interaction with cloud droplets and raindrops. In these studies, it is shown that the wind and rain play an important role in the removal of pollutants from the local regional atmosphere.

To overcome with the shortage of fresh water in various countries in the world (Bulai and Venturino 2016), artificial rain may become an avenue for these countries. In this regard, some modeling studies have been carried out to see the effect of introduction of aerosols in regional atmosphere for making artificial rainfall (Shukla et al. 2010, 2013; Sundar and Sharma 2013; Misra 2016). In particular, Shukla et al. (2010) have proposed a mathematical model for making artificial rain by assuming that two kinds of aerosols are introduced in the atmosphere where water vapors are continuously formed. The first kind of aerosols interact with water vapors to form cloud droplets through the processes of condensation and nucleation. The second kind of aerosols interact with the cloud droplets and convert them into raindrops leading to rainfall. It is shown that the introduction of both kinds of aerosols is very important to get desired amount of rain fall. Latter on Sunder and Sharma (2013) have presented a mathematical model by assuming that cloud droplets are continuously formed in the atmosphere but do not produce sufficient amount of rainfall naturally. It is shown that by introducing external aerosols in the atmosphere, the intensity of rainfall can be increased.

From above, it may be pointed out that physical and chemical properties of atmospheric aerosols have been much studied but a little attention has been paid on the use of aerosols in making rainfall. Keeping this in view, in this paper, we propose a mathematical model dealing with the physicochemical processes occurring in the atmosphere leading to rainfall. In the modeling process, we assume that water vapors are naturally formed in the atmosphere but are not condensed enough to form cloud droplets so as to result into rainfall. By introducing the mixture of aerosols in the localized regional atmosphere, the water vapor may get transformed to form cloud droplets of different sizes through the processes of nucleation, condensation, agglomeration, etc., consequently changing into water drops leading to rainfall.

\section{Mathematical model}

This paper deals with the problem of making artificial rain by introducing conducive mixture of aerosols in a regional atmosphere where vapor clouds are formed continuously. In presence of aerosols, the formation of small size droplets 
is enhanced from vapor clouds through the processes of nucleation and condensation. Further, the transformation of these small size droplets are enhanced by interaction with aerosols to form large size droplets through the processes of condensation, agglomeration etc. In presence of aerosol particles again these large size droplets change to rain drops making rainfall. The kinetics of various interactions follow simple law of mass action.

Let $C_{v}(t)$ be the water vapor density in the atmosphere at any time $t$ and $C_{s d}(t), C_{l d}(t)$ be the number densities (number concentrations of droplets) of small size and large size cloud droplets at any time $t, C_{r}(t)$ and $C_{h}(t)$ be the number density of rain drops and the cumulative concentration of externally introduced conducive aerosols (a mixture of two or more particulate matters) respectively. In the modeling process, it is considered that water vapor phase is continuously formed with the rate $Q_{v}$ (the rate of change of water vapor density assumed to be a constant) and the conducive aerosols are continuously introduced in the atmosphere with the rate $Q_{h}$. The rates of natural depletion of water vapor as well as that of conducive aerosols are assumed to be proportional to their densities respectively. It is also assumed that small size cloud droplets are formed when water vapor phase comes in contact with externally introduced aerosols and thus the density of water vapor decreases. This rate of decrease is assumed to be directly proportional to the density of water vapor as well as that of cumulative density of conducive aerosols (i.e. $C_{v} C_{h}$ ) and hence the density of small cloud droplets increases with the same rate. It is assumed further that the formation of small cloud droplets also takes place from water vapor directly, which is assumed to be proportional to the density of water vapor. The large cloud droplets are formed from small cloud droplets directly, the rate of which is assumed to be proportional to the density of small cloud droplets whereas the rate of formation of large cloud droplets due to the externally introduced aerosols is assumed to be proportional to both the densities of small size cloud droplets as well as the cumulative density of aerosols (i.e. $C_{s d} C_{h}$ ). Further, it is assumed that the natural formation rate of rain drops is proportional to the density of large size cloud droplets, whereas its natural depletion rate is proportional to the density of rain drops. The rate of formation of rain drops by the interaction of large size cloud droplets and aerosols is assumed to be proportional to the density of large size cloud droplets and aerosols (i.e. $\left.C_{l d} C_{h}\right)$. Since aerosols are being used in the formation of small and large size cloud droplets from water vapor as well as in the formation of rain drops from large size cloud droplets, the rate of depletion of cumulative density of aerosols is the same as being used in the nucleation of small size cloud droplets, formation of large size cloud droplets (i.e. $\theta_{1} C_{v} C_{h}, \lambda_{s 1} C_{s d} C_{h}$ ) and formation of raindrops (i.e $\lambda_{l 1} C_{l d} C_{h}$ ). It is also assumed that during rain making, the density of aerosols decreases due to rainfall by impaction, the rate of which is assumed to be proportional to both the number density of rain drops as well as the density of conducive aerosols.

In view of the above assumptions and considerations, the governing equations of the phenomena are proposed as follows:

$$
\begin{aligned}
\frac{d C_{v}}{d t} & =Q_{v}-\theta_{0} C_{v}-\theta_{1} C_{v} C_{h} \\
\frac{d C_{s d}}{d t} & =\lambda_{s} C_{v}-\lambda_{s 0} C_{s d}+\pi_{1} \theta_{1} C_{v} C_{h}-\lambda_{s 1} C_{s d} C_{h} \\
\frac{d C_{l d}}{d t} & =\lambda_{l} C_{s d}-\lambda_{l 0} C_{l d}+\lambda_{s 1} C_{s d} C_{h}-\lambda_{l 1} C_{l d} C_{h} \\
\frac{d C_{r}}{d t} & =r C_{l d}-r_{0} C_{r}+\phi_{1} \lambda_{l 1} C_{l d} C_{h} \\
\frac{d C_{h}}{d t} & =Q_{h}-\delta_{h} C_{h}-\theta_{1} C_{v} C_{h}-\lambda_{s 1} C_{s d} C_{h}-\lambda_{l 1} C_{l d} C_{h}-\lambda_{r} C_{r} C_{h}
\end{aligned}
$$

where $C_{v}(0)>0, \quad C_{s d}(0)>0, \quad C_{l d}(0)>0, \quad C_{r}(0)>0$, $C_{h}(0)>0$.

In the above model (1), the constants $\theta_{0}, \lambda_{s 0}, \lambda_{l 0}, r_{0}$ and $\delta_{h}$ are positive and they represent the natural depletion rate coefficients of water vapor, small size cloud droplets, large size cloud droplets, rain drops and artificially introduced conducive aerosols (mixture) respectively. The coefficients $\lambda_{s} \geq 0, \lambda_{l}>0$ and $r>0$ represent natural formation rate of small cloud droplets from water vapor, large size cloud droplets from small size cloud droplets and rain drops from large size cloud droplets. Thus, it is easy to note here that $\lambda_{s} \leq \theta_{0}, \lambda_{l} \leq \lambda_{s 0}$ and $r \leq \lambda_{l 0}$. The constants $\pi_{1}$ and $\phi_{1}$ are proportionality constants and are positive. The coefficients $\theta_{1}, \lambda_{s 1}, \lambda_{l 1}$ and $\lambda_{r}$ represent rate of transformation between water vapor phase and aerosols, small size cloud droplets and aerosols, large size cloud droplets and aerosols and rain drops and aerosols respectively. These coefficients are also assumed to be positive.

It can be noted here from model (1) that when $\lambda_{s}=0$, the small cloud droplets can not be formed from the vapor phase without its interaction with externally introduced aerosols.

In the following, we analyze the model (1) using stability theory of differential equations. For the solutions of model (1), the region of attraction (Shukla et al. 2010; Misra 2010; Misra et al. 2015) is given by the set:

$$
\begin{aligned}
\Omega= & \left\{\left(C_{v}, C_{s d}, C_{l d}, C_{r}, C_{h}\right): 0 \leq C_{v} \leq \frac{Q_{v}}{\theta_{0}},\right. \\
& 0 \leq C_{s d} \leq R_{s d}, 0 \leq C_{l d} \leq R_{l d}, \\
& \left.0 \leq C_{r} \leq R_{r}, 0 \leq C_{h} \leq \frac{Q_{h}}{\delta_{h}}\right\}
\end{aligned}
$$


which attracts all solutions initiating in the interior of the positive orthant, where $R_{s d}=\frac{Q_{v}}{\lambda_{s 0} \theta_{0}}\left(\lambda_{s}+\frac{\pi_{1} \theta_{1} Q_{h}}{\delta_{h}}\right), R_{l d}=$ $\frac{R_{s d}}{\lambda_{l 0}}\left(\lambda_{l}+\frac{\lambda_{s 1} Q_{h}}{\delta_{h}}\right)$ and $R_{r}=\frac{R_{l d}}{r_{0}}\left(r+\frac{\phi_{1} \lambda_{11} Q_{h}}{\delta_{h}}\right)$.

\section{Equilibrium analysis}

Case-I When the rate of formation of water vapors, i.e. $Q_{v}=0$ and $C_{v}(0)>0$.

In this case when $Q_{v}=0$, the model (1) has only one equilibrium $E_{0}\left(0,0,0,0, \frac{Q_{h}}{\delta_{h}}\right)$. It can be easily seen that the system (1) is dissipative and thus the equilibrium $E_{0}$ is nonlinearly (globally) stable. This implies that initially there would be some rainfall but it decreases to zero asymptotically and thus eventually leading to insufficient rainfall.

Case-II When the rate of formation of water vapors, i.e. $Q_{v}$ is a nonzero constant.

In this case also system (1) has only one nonnegative equilibrium $E^{*}\left(C_{v}^{*}, C_{s d}^{*}, C_{l d}^{*}, C_{r}^{*}, C_{h}^{*}\right)$. This equilibrium can be obtained by solving the following set of algebraic equations:

$Q_{v}-\theta_{0} C_{v}-\theta_{1} C_{v} C_{h}=0$,

$\lambda_{s} C_{v}-\lambda_{s 0} C_{s d}+\pi_{1} \theta_{1} C_{v} C_{h}-\lambda_{s 1} C_{s d} C_{h}=0$,

$\lambda_{l} C_{s d}-\lambda_{l 0} C_{l d}+\lambda_{s 1} C_{s d} C_{h}-\lambda_{l 1} C_{l d} C_{h}=0$,

$r C_{l d}-r_{0} C_{r}+\phi_{1} \lambda_{l 1} C_{l d} C_{h}=0$,

$Q_{h}-\delta_{h} C_{h}-\theta_{1} C_{v} C_{h}-\lambda_{s 1} C_{s d} C_{h}-\lambda_{l 1} C_{l d} C_{h}-\lambda_{r} C_{r} C_{h}=0$.

From Eqs. (3)-(6), we have

$$
\begin{gathered}
C_{v}=\frac{Q_{v}}{\theta_{0}+\theta_{1} C_{h}} \\
C_{s d}=\frac{\left(\lambda_{s}+\pi_{1} \theta_{1} C_{h}\right) C_{v}}{\left(\lambda_{s 0}+\lambda_{s 1} C_{h}\right)} \\
C_{l d}=\frac{\left(\lambda_{l}+\lambda_{s 1} C_{h}\right) C_{s d}}{\left(\lambda_{l 0}+\lambda_{l 1} C_{h}\right)} \\
C_{r}=\frac{\left(r+\phi_{1} \lambda_{l 1} C_{h}\right) C_{l d}}{r_{0}}
\end{gathered}
$$

Using Eqs. (9)-(11) in Eq. (7) and after a simple manipulation, we get the following equation

$$
C_{v}=\frac{\left(Q_{h}-\delta_{h} C_{h}\right)}{C_{h}\left[\theta_{1}+\frac{\left(\lambda_{s}+\pi_{1} \theta_{1} C_{h}\right)}{\left(\lambda_{s 0}+\lambda_{s 1} C_{h}\right)}\left\{\lambda_{s 1}+\frac{\left(\lambda_{l}+\lambda_{s 1} C_{h}\right)}{\left(\lambda_{l 0}+\lambda_{l 1} C_{h}\right)}\left(\lambda_{l 1}+\frac{\lambda_{r}\left(r+\phi_{1} \lambda_{l 1} C_{h}\right)}{r_{0}}\right)\right\}\right]}
$$

Now, we prove the existence of $\left(C_{h}^{*}, C_{v}^{*}\right)$ by plotting the isoclines described by Eqs. (8) and (12) respectively.

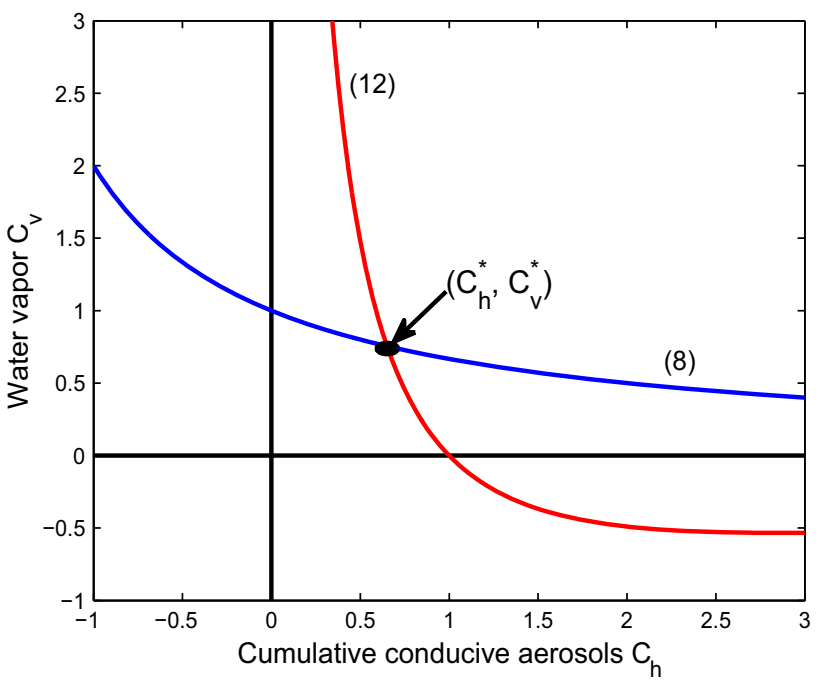

Fig. 1 Plots of the isoclines (8) and (12) in $C_{h}-C_{v}$ - plane, showing the intersection in the positive quadrant at the point $\left(C_{h}^{*}, C_{v}^{*}\right)$ for the parameter values given in (23)

From the isocline given by (8), we note the following:

1. $C_{v}=\frac{Q_{v}}{\theta_{0}}$ at $C_{h}=0$

2. $C_{v} \rightarrow 0$ as $C_{h} \rightarrow \infty$,

3. $\frac{d C_{v}}{d C_{h}}=-\frac{\theta_{1} Q_{v}}{\left(\theta_{0}+\theta_{1} C_{h}\right)^{2}}$, which is negative. Thus $C_{v}$ decreases as $C_{h}$ increases.

Similarly from Eq. (12), we also note the following facts:

1. $C_{v}=0$ at $C_{h}=\frac{Q_{h}}{\delta_{h}}$

2. $C_{v} \rightarrow \infty$ as $C_{h} \rightarrow 0$,

3. $\frac{d C_{v}}{d C_{h}}<0$ inside the open interval $\left(0, \frac{Q_{h}}{\delta_{h}}\right)$ as $\lambda_{s 0} \geq \lambda_{l}$. Thus again $C_{v}$ decreases as $C_{h}$ increases inside the open interval $\left(0, \frac{Q_{h}}{\delta_{h}}\right)$.

Thus the two isoclines described by Eqs. (8) and (12) will always intersect at a unique point $\left(C_{h}{ }^{*}, C_{v}{ }^{*}\right)$ in the interior of first quadrant, see Fig.1.

Using these values of $C_{v}{ }^{*}$ and $C_{h}{ }^{*}$ in Eqs. (9)-(11), we get the equilibrium values of $C_{s d}{ }^{*}, C_{l d}{ }^{*}$ and $C_{r}{ }^{*}$ respectively.

\section{Stability analysis}

The local stability behavior of $E^{*}$ of model (1) is rather complex to study by using Routh-Hurwitz criterion. Hence, we apply the Lyapunov's method to test the local stability of the equilibrium.

Theorem 1 The equilibrium $E^{*}\left(C_{v}^{*}, C_{s d}^{*}, C_{l d}^{*}, C_{r}^{*}, C_{h}^{*}\right)$ is locally asymptotically stable if the following conditions are satisfied: 
$\lambda_{s 1} \theta_{1} C_{v}^{*} C_{h}^{*}<\frac{1}{9} \pi_{1} a^{*}\left(\theta_{0}+\theta_{1} C_{h}^{*}\right)^{2} \min \left[\frac{\lambda_{s 1} a^{*}}{\pi_{1} \theta_{1}^{3} C_{v}^{*} C_{h}^{*}}, \frac{4}{3} \frac{\lambda_{s 0}+\lambda_{s 1} C_{h}^{*}}{\left(\lambda_{s}+\pi_{1} \theta_{1} C_{h}^{*}\right)^{2}}\right]$

$\left(\lambda_{s 1} C_{h}^{*}\right)\left(\lambda_{s 1} C_{s d}^{*}\right)^{2}<\frac{1}{3}\left(\pi_{1} \theta_{1} C_{v}^{*}\right)\left(\lambda_{s 0}+\lambda_{s 1} C_{h}^{*}\right) a^{*}$

$\left(\pi_{1} \theta_{1} \lambda_{l 1} C_{v}^{*}\right)\left(\lambda_{l}+\lambda_{s 1} C_{h}^{*}\right)^{2}<\frac{4}{9} \lambda_{s 1}^{2} C_{s d}^{*}\left(\lambda_{s 0}+\lambda_{s 1} C_{h}^{*}\right)\left(\lambda_{l 0}+\lambda_{l 1} C_{h}^{*}\right)$

$\left(\lambda_{l 1} C_{h}^{*}\right)\left(\lambda_{l 1} C_{l d}^{*}\right)^{2}<\frac{1}{3} \lambda_{s 1} C_{s d}^{*}\left(\lambda_{l 0}+\lambda_{l 1} C_{h}^{*}\right) a^{*}$

$\lambda_{r} \lambda_{s 1} C_{s d}^{*}\left(r+\phi_{1} \lambda_{l 1} C_{h}^{*}\right)^{2}<\frac{4}{3} \phi_{1} r_{0} \lambda_{l 1}^{2} C_{l d}^{*}\left(\lambda_{l 0}+\lambda_{l 1} C_{h}^{*}\right)$

where $a^{*}=\delta_{h}+\theta_{1} C_{v}^{*}+\lambda_{s 1} C_{s d}^{*}+\lambda_{l 1} C_{l d}^{*}+\lambda_{r} C_{r}^{*}$.

For proof see "Appendix 1".

Theorem 2 The equilibrium $E^{*}\left(C_{v}^{*}, C_{s d}^{*}, C_{l d}^{*}, C_{r}^{*}, C_{h}^{*}\right)$ is non-linearly stable inside the region of attraction if the following conditions are satisfied:

$$
\begin{aligned}
& \lambda_{s 1} \theta_{1} C_{v}^{*} C_{h}^{*}<\frac{1}{9} \pi_{1} \theta_{0}^{2} \delta_{h} \min \left[\frac{\lambda_{s 1} \delta_{h}}{\pi_{1} \theta_{1}^{3} C_{v}^{*} C_{h}^{*}}, \frac{4}{3} \frac{\lambda_{s 0}+\lambda_{s 1} C_{h}^{*}}{\left(\lambda_{s}+\pi_{1} \theta_{1} \frac{Q_{h}}{\delta_{h}}\right)^{2}}\right] \\
& \left(\lambda_{s 1} C_{h}^{*}\right)\left(\lambda_{s 1} R_{s d}\right)^{2}<\frac{1}{3}\left(\pi_{1} \theta_{1} \delta_{h} C_{v}^{*}\right)\left(\lambda_{s 0}+\lambda_{s 1} C_{h}^{*}\right) \\
& \left(\pi_{1} \theta_{1} \lambda_{l 1} C_{v}^{*}\right)\left(\lambda_{l}+\lambda_{s 1} \frac{Q_{h}}{\delta_{h}}\right)^{2}<\frac{4}{9} \lambda_{s 1}^{2} C_{s d}^{*}\left(\lambda_{s 0}+\lambda_{s 1} C_{h}^{*}\right) \\
& \left(\lambda_{l 0}+\lambda_{l 1} C_{h}^{*}\right) \\
& \left(\lambda_{l 1} C_{h}^{*}\right)\left(\lambda_{l 1} R_{l d}\right)^{2}<\frac{1}{3} \lambda_{s 1} \delta_{h} C_{s d}^{*}\left(\lambda_{l 0}+\lambda_{l 1} C_{h}^{*}\right) \\
& \lambda_{r} \lambda_{s 1} C_{s d}^{*}\left(r+\phi_{1} \lambda_{l 1} \frac{Q_{h}}{\delta_{h}}\right)^{2}<\frac{4}{3} \phi_{1} r_{0} \lambda_{l 1}^{2} C_{l d}^{*}\left(\lambda_{l 0}+\lambda_{l 1} C_{h}^{*}\right)
\end{aligned}
$$

For proof of the theorem see "Appendix 2".

Remark 1 When $\theta_{1}=0$, it is noted that local stability conditions (13) and (15), non-linear stability conditions (18) and (20) are automatically satisfied but local stability condition (14) and global (non-linear) stability condition (19) are never satisfied. This implies that the process of interaction of aerosols with water vapor is destabilizing in nature.

Remark 2 When $\lambda_{s 1}=0$, it may also be noted that local stability conditions (14) and (17), non-linear stability conditions (19) and (22) are automatically satisfied but local stability condition (16) and global (non-linear) stability condition (21) are not satisfied. This implies that the process of interaction of aerosols with cloud droplets has destabilizing effect on the system.

\section{Numerical simulation}

To check the feasibility of our analysis regarding stability conditions and to see effect of other parameters on the rainfall, we have conducted some numerical computation by choosing the following set of values of the parameters in model (1).

$$
\begin{aligned}
Q_{v} & =1.0, \theta_{0}=1.0, \theta_{1}=0.5, \lambda_{s}=0.1, \lambda_{s 0}=1.0, \pi_{1} \\
& =1, \lambda_{s 1}=0.5, \lambda_{l}=0.1 \\
\lambda_{l 0} & =1.0, \lambda_{l 1}=0.3, r=0.1, r_{0}=0.02 \\
\phi_{1} & =1, Q_{h}=1.0, \delta_{h}=1.0, \lambda_{r}=0.01
\end{aligned}
$$

It may be checked that for the above set of parameter values, the local stability conditions (13)-(17) and nonlinear stability conditions (18)-(22) are satisfied. For the above set of data, we have calculated the equilibrium $E^{*}\left(C_{v}^{*}, C_{s d}^{*}, C_{l d}^{*}, C_{r}^{*}, C_{h}^{*}\right)$. The values of $C_{v}^{*}, C_{s d}^{*}, C_{l d}^{*}, C_{r}^{*}$ and $C_{h}^{*}$ are given as follows:

$C_{v}^{*}=0.7545, C_{s d}^{*}=0.2422, C_{l d}^{*}=0.0862, C_{r}^{*}=1.2719$, $C_{h}^{*}=0.6507$.

In this case, we have numerically calculated eigenvalues of Jacobian matrix using above data. The eigenvalues of the Jacobian matrix corresponding to the equilibrium $E^{*}$ are obtained as, $-1.5822,-0.9565,-0.0203,-1.4219+$ $0.2419 i$ and $-1.4219-0.2419 i$. It may be noted that these are either negative or have negative real part, hence the equilibrium $E^{*}$ is locally stable.

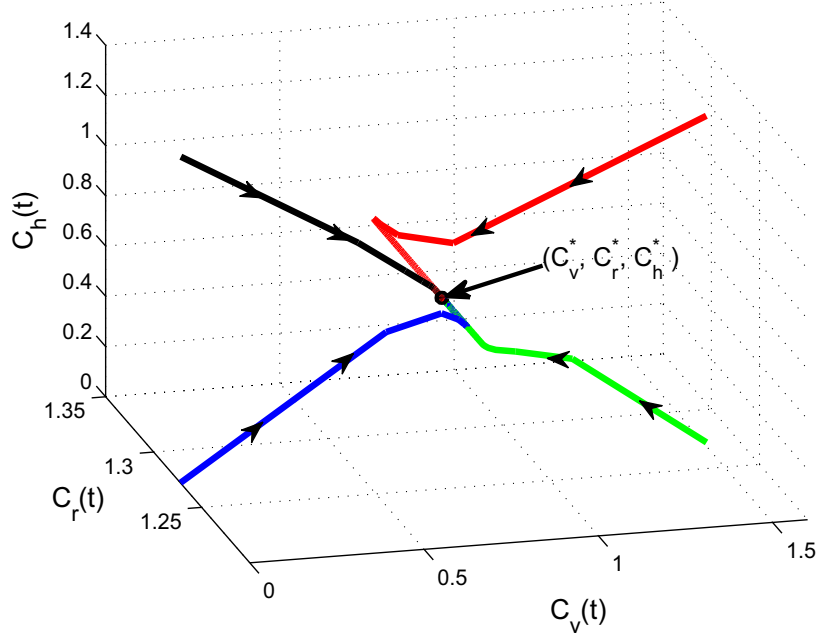

Fig. 2 Plots of the solution trajectories of model system (1) initiating inside the region of attraction. All the trajectories are approaching towards the equilibrium values showing the global stability in $C_{v}-C_{r}-C_{h^{-}}$space 
Fig. 3 Variation in the (a) density of small size cloud droplets $C_{s d}$, (b) density of large size cloud droplets $C_{l d}$,

(c) density of raindrops $C_{r}$ and (d) cumulative concentration of aerosols $C_{h}$, w.r.t. time $t$ for different values of rate of formation of water vapors in the atmosphere $Q_{v}$, keeping other parameter values same as given in (23)

Fig. 4 Variation in the

(a) density of water vapors $C_{v}$, (b) density of small size cloud droplets $C_{s d}$, (c) density of large size cloud droplets $C_{l d}$ and (d) density of raindrops $C_{r}$ w.r.t. time $t$ for different values of rate of introduction of aerosols in the atmosphere $Q_{h}$, keeping other parameter values same as given

in (23) trajectories for $C_{v}, C_{r}$ and $C_{h}$ with different initial starts have been drawn in Fig. 2. From this figure, it is clear that
Further, for the above set of parameters, the solution

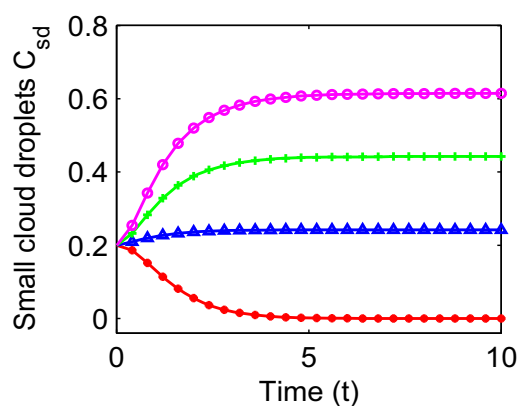

(a)

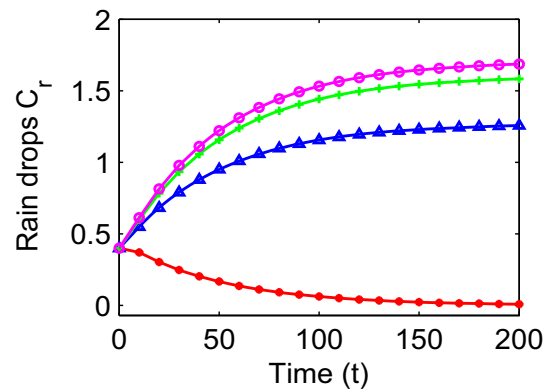

(c)

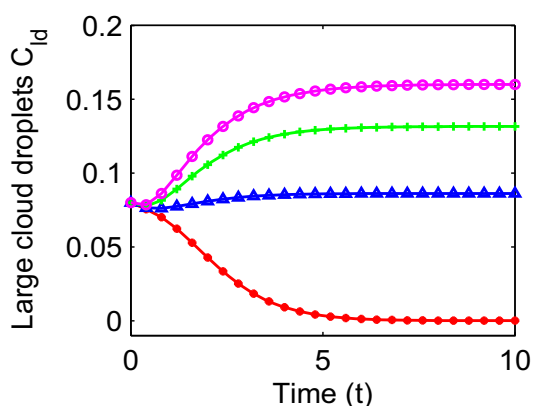

(b)

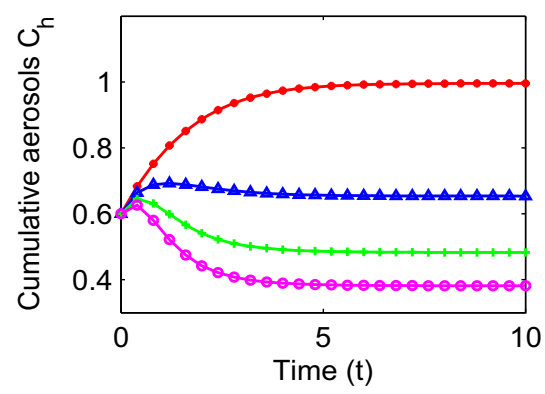

(d)

$$
\longrightarrow Q_{v}=0, \quad \longrightarrow Q_{v}=1, \quad \longrightarrow Q_{v}=2, \quad \longrightarrow Q_{v}=3
$$

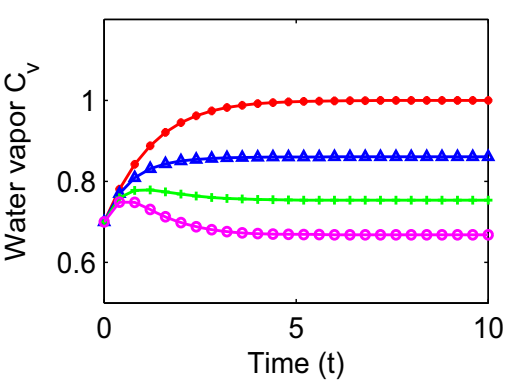

(a)

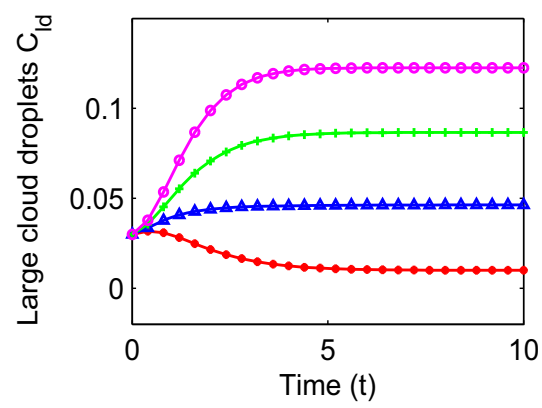

(c)

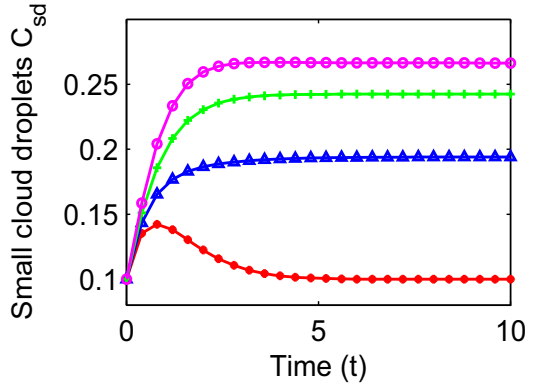

(b)

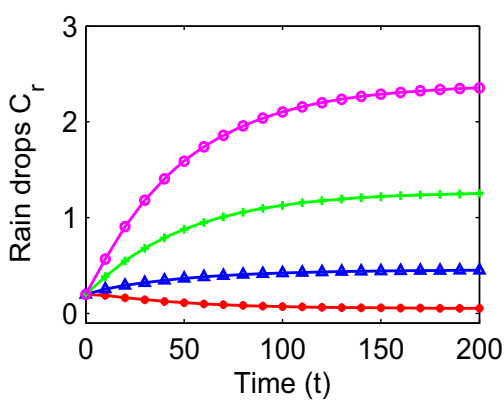

(d)

$$
\longrightarrow Q_{h}=0, \longrightarrow Q_{h}=0.5, \longrightarrow Q_{h}=1, \longrightarrow Q_{h}=1.5
$$

all trajectories are being attracted by equilibrium values, which indicates the non-linear stability of $\left(C_{v}^{*}, C_{r}^{*}, C_{h}^{*}\right)$ in $C_{v}-C_{r}-C_{h}$ space. 
Fig. 5 Variation in the equilibrium values of (a) density of water vapors $C_{v}$, (b) density of small size cloud droplets $C_{s d}$, (c) density of large size cloud droplets $C_{l d}$,

(d) density of raindrops $C_{r}$ and (e) cumulative concentration of aerosols $C_{h}$ w.r.t. $Q_{v}$ and $Q_{h}$. Rest of the parameter values are the same as in (23)

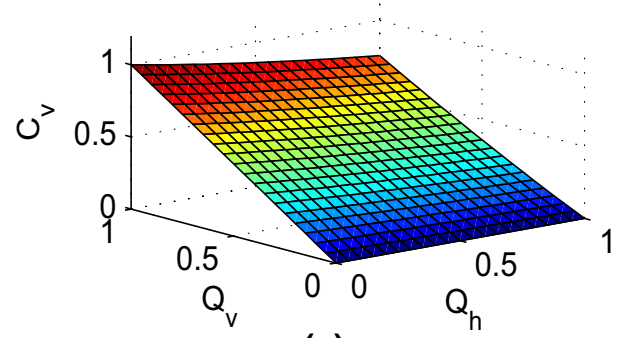

(a)

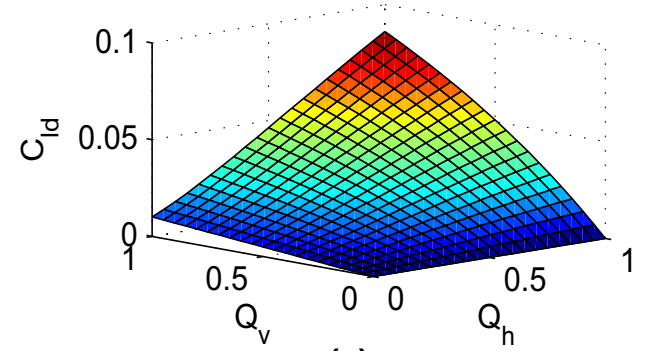

(c)

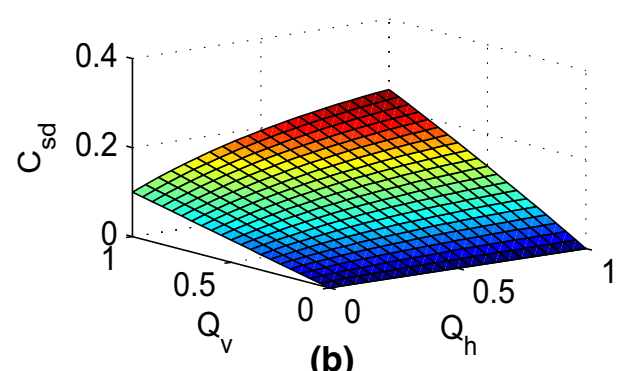

(b)

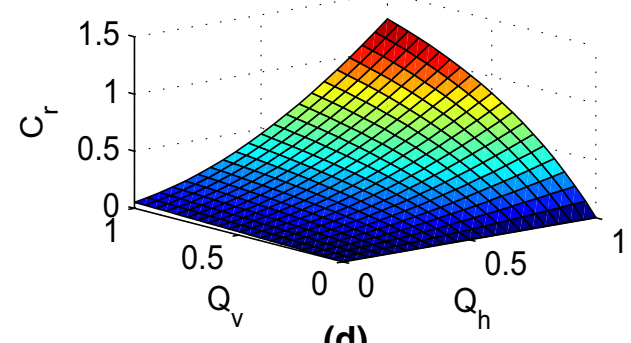

(d)

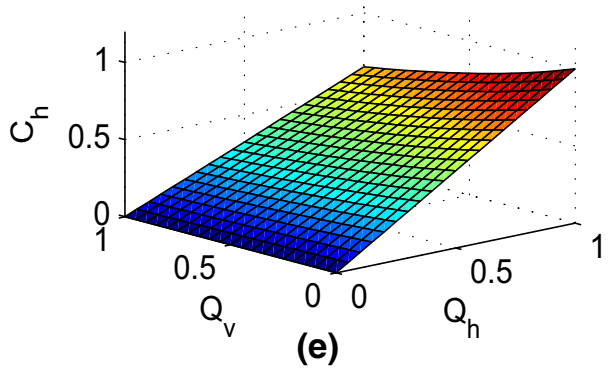

The effect of rate of change of water vapor $Q_{v}$ in the atmosphere on the variables $C_{s d}, C_{l d}, C_{r}$ and $C_{h}$ is presented in the Fig.3a-d. Here, the values of other parameters are the same as given in (23) except $Q_{v}$. From these figures, it is clear that as $Q_{v}$ increases, the equilibrium level of densities of cloud droplets (small and large) and rain drops increases whereas the equilibrium level of cumulative concentration of aerosols decreases. From the Fig. 3a-c, we also see that if the water vapors are not continuously formed in the atmosphere i.e $Q_{v}=0$, then the density of small size cloud droplets, large size cloud droplets and density of rain drops tend to zero after a short period of time. It means initially there will be some rain due to the presence of initial amount of water vapor in the atmosphere but it will stop after some time. From Fig. 3d, we note that if $Q_{v}=0$ then the cumulative concentration of aerosols tends to its maximum value i.e. $Q_{h} / \delta_{h}$.

The effect of rate of introduction of the mixture of aerosols $Q_{h}$ in the atmosphere on the variables $C_{v}, C_{s d}, C_{l d}$ and $C_{r}$ is shown in Fig.4a-d, by keeping the values of other parameters same as given in (23). From these figures, it is clear that as $Q_{h}$ increases, the equilibrium level of densities of cloud droplets (small and large) and rain drops increases whereas the equilibrium level of density of water vapors decreases. From this figure, it is also clear that for $Q_{h}=0$, the density of water vapors reaches to its maximum value; however their will be a small amount of rain due to the natural conversion of water vapors into cloud droplets and cloud droplets into rain drops.

In Figs. 5 and 6, we have shown the variations in the equilibrium densities of water vapors, cloud droplets (small and large) and rain drops and the equilibrium level of cumulative concentration of aerosols w.r.t. $Q_{v}, Q_{h}, \theta_{1}$ and $\lambda_{l 1}$ for the set of parameter values given in (23). We have plotted all the five variables of the model system (1) by varying two parameters at a time viz. $\left(Q_{v}, Q_{h}\right)$ and $\left(\theta_{1}, \lambda_{l 1}\right)$, in the interval $[0,1] \times[0,1]$. From the Fig. 5 , it is easy to see that for $Q_{v}=0$, the equilibrium densities of water vapors, clouds (small and large size), rain drops are zero whatsoever be the value of $Q_{h}$; however in this case the equilibrium level of cumulative concentration is at its maximum value. Further for $Q_{h}=0$, on increasing the value of $Q_{v}$, the equilibrium densities of $C_{v}, C_{s d}, C_{l d}$ and $C_{r}$ increase and in this case a little amount of rain can be observed due to natural processes of condensation and nucleation. Increasing both $Q_{v}$ and $Q_{h}$ leads to increase in 
Fig. 6 Variation in the equilibrium values of (a) density of water vapors $C_{v}$, (b) density of small size cloud droplets $C_{s d}$, (c) density of large size cloud droplets $C_{l d}$,

(d) density of raindrops $C_{r}$ and (e) cumulative concentration of aerosols $C_{h}$ w.r.t. $\theta_{1}$ and $\lambda_{l 1}$. Rest of the parameter values are the same as in (23)

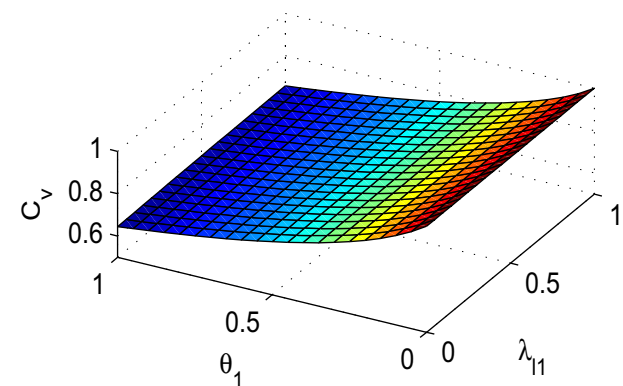

(a)

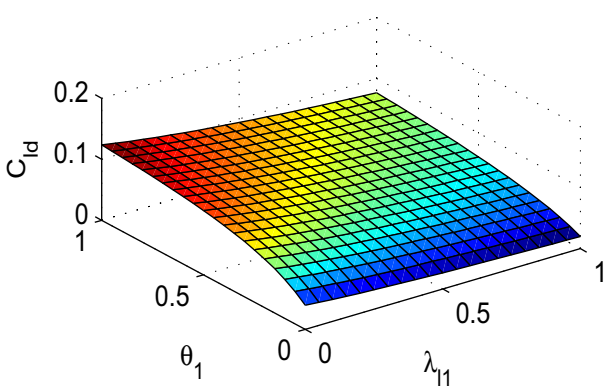

(c)

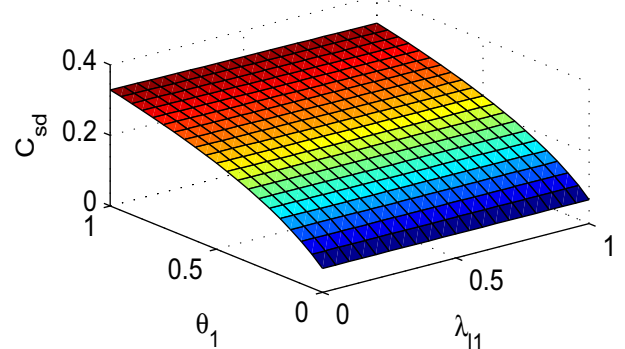

(b)

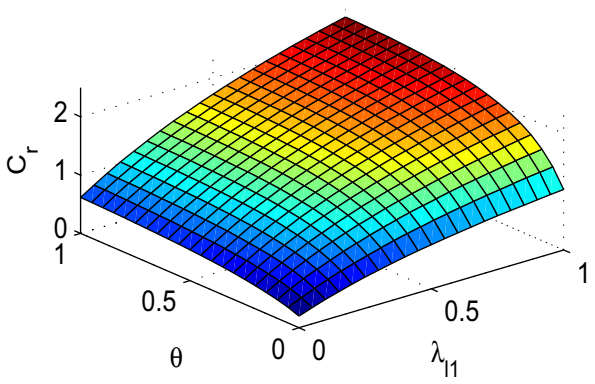

(d)

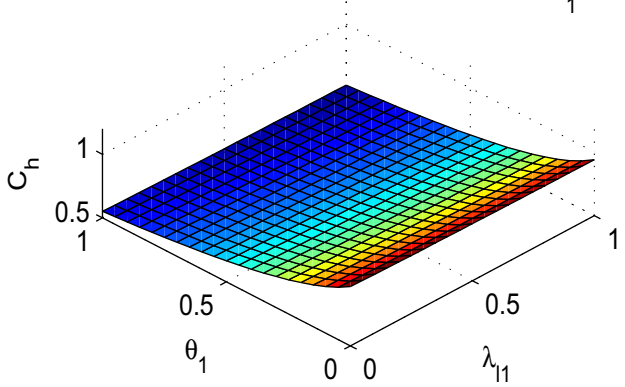

(e)

the equilibrium densities of cloud droplets (small and large sizes) and rain drops on the diagonal, showing the importance of introduction of aerosols in the atmosphere. It is apparent from the Fig. 6 that increasing the values of $\theta_{1}$ leads to increase in the equilibrium values of densities of cloud droplets (small and large sizes) and rain drops but decrease in the density of water vapors and cumulative concentration of aerosols. From this figure, it is also clear that increase in the values of the parameter $\lambda_{l 1}$ leads to decrease in the large size cloud droplets and much increase in rainfall, showing the importance of introduction of mixture of aerosols.

From these figures, we may easily note that the continuous formation of water vapors and the continuous introduction of mixture of aerosols, which enhances the condensation of water vapors to form cloud droplets and changing cloud droplets into rain drops are necessary to get the desired rainfall. This implies that for continuous rainfall, not only the formation of water vapor is necessary but continuous introduction of aerosols is also essential.

\section{Conclusion}

In this paper, a non-linear model for rain making is proposed and analyzed by introducing conducive aerosols in the localized regional atmosphere, where water vapors are continuously formed at a constant rate. It is assumed that conducive aerosol particles interact with water vapors in the atmosphere making the water vapors to become cloud droplets of small and large sizes through the processes of nucleation, condensation, agglomeration, etc., leading to rainfall. It is assumed that small size cloud droplets are formed directly from water vapors as well as by the interactions of water vapors and aerosol particles. Similarly, the large size cloud droplets are formed from small size cloud droplets directly as well as due to interactions of small size cloud droplets and aerosols. It is assumed further that rainfall is made from large size cloud droplets directly as well as due to interactions of large size cloud droplets with aerosol particles.

The analysis reveals that for the proposed model only one equilibrium is feasible. The sufficient conditions for the local as well as global stability of this equilibrium are 
obtained. It is found that the system may loose its stability on increasing the values of the rate of interactions of aerosols with water vapors and cloud droplets.

The results obtained from numerical simulations clearly indicate that for continuous rainfall, the water vapors must be continuously formed in the atmosphere. From Fig. 6, it is easy to see that for desired amount of rainfall, the aerosols which are introduced in the atmosphere must be a mixture of aerosols. This mixture must contain the aerosols which increases the process of condensation of water vapors to form cloud droplets and the aerosols which increases the process of nucleation and agglomeration to change the large size cloud droplets into the raindrops. From Fig. 6d, it is also apparent that the introduction of only one kind of aerosols, either which increases the condensation process of water vapors to form cloud droplets or which increases the rate of formation of rain drops from large size cloud droplets, leads to a little increment in the rainfall. However, a mixture of aerosols accounts for a significant change in the amount of rainfall on the diagonal. Apart from the introduction of mixture of aerosols, from Fig. 5d, it may be noted that the amount of rainfall also depends on the rate of introduction of aerosols in the atmosphere.

\section{Appendix 1: Proof of Theorem 1}

Linearizing the system (1) about $E^{*}$ by using the following transformations

$$
\begin{aligned}
& C_{v}=C_{v}^{*}+C_{v 1}, C_{s d}=C_{s d}^{*}+C_{s d 1}, \\
& C_{l d}=C_{l d}^{*}+C_{l d 1}, C_{r}=C_{r}^{*}+C_{r 1}, C_{h}=C_{h}^{*}+C_{h 1} .
\end{aligned}
$$

Now using the following positive definite function

$$
V=\frac{1}{2}\left(C_{v 1}^{2}+m_{1} C_{s d 1}^{2}+m_{2} C_{l d 1}^{2}+m_{3} C_{r 1}^{2}+m_{4} C_{h 1}^{2}\right)
$$

(where $m_{1}, m_{2}, m_{3}, m_{4}$ are some positive constants to be chosen appropriately).

Differentiating above equation with respect to $t$ along the solutions of linearized system of (1), we get

$$
\begin{aligned}
\frac{d V}{d t}= & -\left(\theta_{0}+\theta_{1} C_{h}^{*}\right) C_{v 1}^{2}-m_{1}\left(\lambda_{s 0}+\lambda_{s 1} C_{h}^{*}\right) C_{s d 1}^{2}-m_{2}\left(\lambda_{l 0}\right. \\
& \left.+\lambda_{l 1} C_{h}^{*}\right) C_{l d 1}^{2}-m_{3} r_{0} C_{r 1}^{2}-m_{4} a^{*} C_{h 1}^{2} \\
& +C_{v 1} C_{h 1}\left[-\theta_{1} C_{v}^{*}-m_{4} \theta_{1} C_{h}^{*}\right]+C_{v 1} C_{s d 1}\left[m _ { 1 } \left(\lambda_{s}\right.\right. \\
& \left.\left.+\pi_{1} \theta_{1} C_{h}^{*}\right)\right]+C_{s d 1} C_{h 1}\left[m_{1}\left(\pi_{1} \theta_{1} C_{v}^{*}-\lambda_{s 1} C_{s d}^{*}\right)\right. \\
& \left.-m_{4} \lambda_{s 1} C_{h}^{*}\right]+C_{s d 1} C_{l d 1}\left[m_{2}\left(\lambda_{l}+\lambda_{s 1} C_{h}^{*}\right)\right] \\
& +C_{l d 1} C_{h 1}\left[m_{2}\left(\lambda_{s 1} C_{s d}^{*}-\lambda_{l l} C_{l d}^{*}\right)-m_{4} \lambda_{l 1} C_{h}^{*}\right] \\
& +C_{l d 1} C_{r 1}\left[m_{3}\left(r+\phi_{1} \lambda_{l 1} C_{h}^{*}\right)\right]+C_{r 1} C_{h 1}\left[m_{3} \phi_{1} \lambda_{l 1} C_{l d}^{*}\right. \\
& \left.-m_{4} \lambda_{r} C_{h}^{*}\right]
\end{aligned}
$$

Choosing $\quad m_{2}=m_{1} \frac{\pi_{1} \theta_{1} \lambda_{l 1} C_{v}^{*}}{\lambda_{s 1}^{2} C_{s d}^{*}}, \quad m_{3}=m_{1} \frac{\pi_{1} \theta_{1} \lambda_{r} C_{v}^{*}}{\phi_{1} \lambda_{s 1} \lambda_{l 1} C_{l d}^{*}} \quad$ and $m_{4}=m_{1} \frac{\pi_{1} \theta_{1} C_{v}^{*}}{\lambda_{s 1} C_{h}^{*}}, \frac{d V}{d t}$ is simplified as:

$$
\begin{aligned}
\frac{d V}{d t}= & -\left(\theta_{0}+\theta_{1} C_{h}^{*}\right) C_{v 1}^{2}-m_{1}\left(\lambda_{s 0}+\lambda_{s 1} C_{h}^{*}\right) C_{s d 1}^{2} \\
& -m_{1} \frac{\pi_{1} \theta_{1} \lambda_{l 1} C_{v}^{*}}{\lambda_{s 1}^{2} C_{s d}^{*}}\left(\lambda_{l 0}+\lambda_{l 1} C_{h}^{*}\right) C_{l d 1}^{2} \\
& -m_{1} \frac{\pi_{1} \theta_{1} \lambda_{r} r_{0} C_{v}^{*}}{\phi_{1} \lambda_{s 1} \lambda_{l 1} C_{l d}^{*}} C_{r 1}^{2}-m_{1} \frac{\pi_{1} \theta_{1} C_{v}^{*} a^{*}}{\lambda_{s 1} C_{h}^{*}} C_{h 1}^{2} \\
& +C_{v 1} C_{h 1}\left[-\theta_{1} C_{v}^{*}\right]+C_{v 1} C_{h 1}\left[-m_{1} \frac{\pi_{1} \theta_{1}^{2} C_{v}^{*}}{\lambda_{s 1}}\right] \\
& +C_{v 1} C_{s d 1}\left[m_{1}\left(\lambda_{s}+\pi_{1} \theta_{1} C_{h}^{*}\right)\right] \\
& +C_{s d 1} C_{h 1}\left[-m_{1} \lambda_{s 1} C_{s d}^{*}\right] \\
& +C_{s d 1} C_{l d 1}\left[m_{1} \frac{\pi_{1} \theta_{1} \lambda_{l 1} C_{v}^{*}}{\lambda_{s 1}^{2} C_{s d}^{*}}\left(\lambda_{l}+\lambda_{s 1} C_{h}^{*}\right)\right] \\
& +C_{l d 1} C_{h 1}\left[-m_{1} \frac{\pi_{1} \theta_{1} \lambda_{l 1}^{2} C_{v}^{*} C_{l d}^{*}}{\lambda_{s 1}^{2} C_{s d}^{*}}\right] \\
& +C_{l d 1} C_{r 1}\left[m_{1} \frac{\pi_{1} \theta_{1} \lambda_{r} C_{v}^{*}\left(r+\phi_{1} \lambda_{l 1} C_{h}^{*}\right)}{\phi_{1} \lambda_{s 1} \lambda_{l 1} C_{l d}^{*}}\right]
\end{aligned}
$$

Now $\frac{d V}{d t}$ will be negative definite provided the conditions (13)-(17) are satisfied.

\section{Appendix 2: Proof of Theorem 2}

To prove this theorem we consider the following positive definite function about $E^{*}$,

$$
\begin{aligned}
U= & \frac{1}{2}\left[\left(C_{v}-C_{v}^{*}\right)^{2}+k_{1}\left(C_{s d}-C_{s d}^{*}\right)^{2}\right. \\
& \left.+k_{2}\left(C_{l d}-C_{l d}^{*}\right)^{2}+k_{3}\left(C_{r}-C_{r}^{*}\right)^{2}+k_{4}\left(C_{h}-C_{h}^{*}\right)^{2}\right]
\end{aligned}
$$

(where $k_{1}, k_{2}, k_{3}$ and $k_{4}$ are some positive constants to be chosen appropriately).

Differentiating above equation with respect to $t$ along the solutions of system (1) and after a simple algebraic manipulation we get,

$$
\begin{aligned}
\frac{d U}{d t=} & -k_{4}\left(\theta_{1} C_{v}+\lambda_{s 1} C_{s d}+\lambda_{l 1} C_{l d}+\lambda_{r} C_{r}\right)\left(C_{h}-C_{h}^{*}\right)^{2} \\
& -\left(\theta_{0}+\theta_{1} C_{h}^{*}\right)\left(C_{v}-C_{v}^{*}\right)^{2}-k_{1}\left(\lambda_{s 0}+\lambda_{s 1} C_{h}^{*}\right)\left(C_{s d}\right. \\
& \left.-C_{s d}^{*}\right)^{2}-k_{2}\left(\lambda_{l 0}+\lambda_{l 1} C_{h}^{*}\right)\left(C_{l d}-C_{l d}^{*}\right)^{2}-k_{3} r_{0}\left(C_{r}\right. \\
& \left.-C_{r}^{*}\right)^{2}-k_{4} \delta_{h}\left(C_{h}-C_{h}^{*}\right)^{2}+\left(C_{v}-C_{v}^{*}\right)\left(C_{h}\right. \\
& \left.-C_{h}^{*}\right)\left[-\theta_{1} C_{v}-k_{4} \theta_{1} C_{h}^{*}\right]+\left(C_{v}-C_{v}^{*}\right)\left(C_{s d}\right. \\
& \left.-C_{s d}^{*}\right)\left[k_{1}\left(\lambda_{s}+\pi_{1} \theta_{1} C_{h}\right)\right]+\left(C_{s d}-C_{s d}^{*}\right)\left(C_{h}\right. \\
& \left.-C_{h}^{*}\right)\left[k_{1}\left(\pi_{1} \theta_{1} C_{v}^{*}-\lambda_{s 1} C_{s d}\right)-k_{4} \lambda_{s 1} C_{h}^{*}\right]+\left(C_{s d}\right. \\
& \left.-C_{s d}^{*}\right)\left(C_{l d}-C_{d d}^{*}\right)\left[k_{2}\left(\lambda_{l}+\lambda_{s 1} C_{h}\right)\right]+\left(C_{l d}\right. \\
& \left.-C_{l d}^{*}\right)\left(C_{h}-C_{h}^{*}\right)\left[k_{2}\left(\lambda_{s 1} C_{s d}^{*}-\lambda_{l 1} C_{l d}\right)-k_{4} \lambda_{l 1} C_{h}^{*}\right] \\
& +\left(C_{l d}-C_{l d}^{*}\right)\left(C_{r}-C_{r}^{*}\right)\left[k_{3}\left(r+\phi_{1} \lambda_{l 1} C_{h}\right)\right]+\left(C_{r}\right. \\
& \left.-C_{r}^{*}\right)\left(C_{h}-C_{h}^{*}\right)\left[k_{3} \phi_{1} \lambda_{l 1} C_{l d}^{*}-k_{4} \lambda_{r} C_{h}^{*}\right]
\end{aligned}
$$

Choosing $\quad k_{2}=k_{1} \frac{\pi_{1} \theta_{1} \lambda_{l l} C_{v}^{*}}{\lambda_{s 1}^{2} C_{s d}^{*}}, \quad k_{3}=k_{1} \frac{\pi_{1} \theta_{1} \lambda_{r} C_{v}^{*}}{\phi_{1} \lambda_{s 1} \lambda_{l 1} C_{l d}^{*}} \quad$ and $k_{4}=k_{1} \frac{\pi_{1} \theta_{1} C_{v}^{*}}{\lambda_{s 1} C_{h}^{*}}, \frac{d U}{d t}$ is simplified as 


$$
\begin{aligned}
\frac{d U}{d t}= & -k_{1} \frac{\pi_{1} \theta_{1} \lambda_{l l} C_{v}^{*}}{\lambda_{s 1}^{2} C_{s d}^{*}}\left(\theta_{1} C_{v}+\lambda_{s 1} C_{s d}+\lambda_{l l} C_{l d}+\lambda_{r} C_{r}\right)\left(C_{h}-C_{h}^{*}\right)^{2} \\
& -\left(\theta_{0}+\theta_{1} C_{h}^{*}\right)\left(C_{v}-C_{v}^{*}\right)^{2}-k_{1}\left(\lambda_{s 0}+\lambda_{s 1} C_{h}^{*}\right)\left(C_{s d}-C_{s d}^{*}\right)^{2} \\
& -k_{1} \frac{\pi_{1} \theta_{1} \lambda_{l l} C_{v}^{*}}{\lambda_{s 1}^{2} C_{s d}^{*}}\left(\lambda_{l 0}+\lambda_{l l} C_{h}^{*}\right)\left(C_{l d}-C_{l d}^{*}\right)^{2}-k_{1} \frac{\pi_{1} \theta_{1} \lambda_{r} r_{0} C_{v}^{*}}{\phi_{1} \lambda_{s 1} \lambda_{l 1} C_{l d}^{*}}\left(C_{r}-C_{r}^{*}\right)^{2} \\
& -k_{1} \frac{\pi_{1} \theta_{1} \delta_{h} C_{v}^{*}}{\lambda_{s 1} C_{h}^{*}}\left(C_{h}-C_{h}^{*}\right)^{2} \\
& +\left(C_{v}-C_{v}^{*}\right)\left(C_{h}-C_{h}^{*}\right)\left[-\theta_{1} C_{v}\right] \\
& +\left(C_{v}-C_{v}^{*}\right)\left(C_{h}-C_{h}^{*}\right)\left[-k_{1} \frac{\pi_{1} \theta_{1}^{2} C_{v}^{*}}{\lambda_{s 1}}\right] \\
& +\left(C_{v}-C_{v}^{*}\right)\left(C_{s d}-C_{s d}^{*}\right)\left[k_{1}\left(\lambda_{s}+\pi_{1} \theta_{1} C_{h}\right)\right] \\
& +\left(C_{s d}-C_{s d}^{*}\right)\left(C_{h}-C_{h}^{*}\right)\left[-k_{1} \lambda_{s 1} C_{s d}\right] \\
& +\left(C_{s d}-C_{s d}^{*}\right)\left(C_{l d}-C_{l d}^{*}\right)\left[k_{1} \frac{\pi_{1} \theta_{1} \lambda_{l l} C_{v}^{*}}{\lambda_{s 1}^{2} C_{s d}^{*}}\left(\lambda_{l}+\lambda_{s 1} C_{h}\right)\right] \\
& +\left(C_{l d}-C_{l d}^{*}\right)\left(C_{h}-C_{h}^{*}\right)\left[-k_{1} \frac{\pi_{1} \theta_{1} \lambda_{l l}^{2} C_{v}^{*}}{\lambda_{s 1}^{2} C_{s d}^{*}} C_{l d}\right] \\
& +\left(C_{l d}-C_{l d}^{*}\right)\left(C_{r}-C_{r}^{*}\right)\left[k_{1} \frac{\pi_{1} \theta_{1} \lambda_{r} C_{v}^{*}}{\phi_{1} \lambda_{s 1} \lambda_{l 1} C_{l d}^{*}}\left(r+\phi_{1} \lambda_{l 1} C_{h}\right)\right]
\end{aligned}
$$

Now $\frac{d U}{d t}$ will be negative definite inside the region of attraction provided the following conditions are satisfied:

$$
\begin{aligned}
& {\left[\theta_{1} \frac{Q_{v}}{\theta_{0}}\right]^{2}<\frac{1}{3} k_{1} \delta_{h} \frac{\pi_{1} \theta_{1} C_{v}^{*}}{\lambda_{s 1} C_{h}^{*}}\left(\theta_{0}+\theta_{1} C_{h}^{*}\right)} \\
& k_{1}\left[\frac{\pi_{1} \theta_{1}^{2} C_{v}^{*}}{\lambda_{s 1}}\right]^{2}<\frac{1}{3} \delta_{h} \frac{\pi_{1} \theta_{1} C_{v}^{*}}{\lambda_{s 1} C_{h}^{*}}\left(\theta_{0}+\theta_{1} C_{h}^{*}\right) \\
& k_{1}\left[\lambda_{s}+\pi_{1} \theta_{1} \frac{Q_{h}}{\delta_{h}}\right]^{2}<\frac{4}{9}\left(\theta_{0}+\theta_{1} C_{h}^{*}\right)\left(\lambda_{s 0}+\lambda_{s 1} C_{h}^{*}\right) \\
& \left(\lambda_{s 1} R_{s d}\right)^{2}<\frac{1}{3} \delta_{h} \frac{\pi_{1} \theta_{1} C_{v}^{*}}{\lambda_{s 1} C_{h}^{*}}\left(\lambda_{s 0}+\lambda_{s 1} C_{h}^{*}\right) \\
& \frac{\pi_{1} \theta_{1} \lambda_{l 1} C_{v}^{*}}{\lambda_{s 1}^{2} C_{s d}^{*}}\left[\lambda_{l}+\frac{\lambda_{s 1} Q_{h}}{\delta_{h}}\right]^{2}<\frac{4}{9}\left(\lambda_{s 0}+\lambda_{s 1} C_{h}^{*}\right)\left(\lambda_{l 0}+\lambda_{l 1} C_{h}^{*}\right) \\
& \frac{\lambda_{l 1} C_{h}^{*}}{\lambda_{s 1} C_{s d}^{*}}\left(\lambda_{l 1} R_{l d}\right)^{2}<\frac{1}{3} \delta_{h}\left(\lambda_{l 0}+\lambda_{l 1} C_{h}^{*}\right) \\
& \frac{\lambda_{r}}{\phi_{1} \lambda_{l 1} C_{l d}^{*}}\left[r+\frac{\phi_{1} \lambda_{l 1} Q_{h}}{\delta_{h}}\right]^{2}<\frac{4}{3} \frac{\lambda_{l 1}}{\lambda_{s 1} C_{s d}^{*}} r_{0}\left(\lambda_{l 0}+\lambda_{l 1} C_{h}^{*}\right)
\end{aligned}
$$

From above inequalities (30), (31) and (32), we may choose $k_{1}>0$ if the following condition is satisfied:

$$
\lambda_{s 1} \theta_{1} C_{\nu}^{*} C_{h}^{*}<\frac{1}{9} \pi_{1} \theta_{0}^{2} \delta_{h} \min \left[\frac{\lambda_{s 1} \delta_{h}}{\pi_{1} \theta_{1}^{3} C_{v}^{*} C_{h}^{*}}, \frac{4}{3} \frac{\lambda_{s 0}+\lambda_{s 1} C_{h}^{*}}{\left(\lambda_{s}+\pi_{1} \theta_{1} \frac{Q_{h}}{\delta_{h}}\right)^{2}}\right]
$$

Here we have used the fact that $\left(\theta_{0}+\theta_{1} C_{h}^{*}\right)=Q_{v} / C_{v}^{*}$. Thus, the system (1) is globally stable inside the region of attraction if the conditions (18)-(22) are satisfied.

\section{References}

Agarwal M, Tandon A (2010) Modeling of the urban heat island in the form of mesoscale wind and of its effect on air pollution dispersal. Appl Math Model 34:2520-2530

Bower KN, Choularton TW, Gallagher MW, Colvile RN, Wells M, Beswick KM, Wiedensohler A, Hansson HC, Svenningsson B, Swietlicki E, Wendisch M, Berner A, Kruisz C, Laj P, Facchini MC, Fuzzi S, Bizjak M, Dollard G, Jones J, Acker K, Wieprecht W, Preiss M, Sutton MA, Hargreaves KJ, Storeton-West RL, Cape JN, Arends BG (1997) Observations and modeling of the processing of aerosol by a hill cap cloud. Atmos Environ $31: 2524-2543$

Bulai IM, Venturino E (2016) Biodegradation of organic pollutants in a water body. J Math Chem 54:1387-1403

Coffman DJ, Hegg DA (1995) A preliminary study of the effect of ammonia on particle nucleation in the marine boundary layer. J Geophs Res 100:7147-7160

Easter RC, Hobbs PV (1974) The formation of sulphate and the enhancement of cloud condensation nuclei in clouds. J Atmos Sci 31:1586-1591

Flossmann AI, Pruppacher HR, Topolian JH (1987) A theoretical study of the wet removal of atmospheric pollutants, Part II: The uptake and redistribution of $\left(\mathrm{NH}_{4}\right)_{2} \mathrm{SO}_{4}$ particles and $\mathrm{SO}_{2}$ gas simultaneously scavenged by growing cloud drops. J Atmos Sci 44:2912-2923

Finlayson-Pitts BJ, Pitts JN Jr (2000) Chemistry of the upper and lower atmosphere-theory, experiments and applications. Academic Press, San Diego

Hegg DA (1990) Heterogeneous production of cloud condensation nuclei in the marine atmosphere: Geophys. Res Lett $17: 2165-2168$

Hegg DA (2001) The impact of clouds on aerosol populations. IGACtivities No. 23

Houghton JT, Ding Y, Griggs DJ, Noguer M, van der Linden PJ, Dai X, Maskell K, Johnson CA (2001) Climate change: the scientific basis (contribution of working group $\mathrm{i}$ to the third assessment report of the intergovernmental panel on climate change). Cambridge University Press, Cambridge

Hudson JG, Frisbie PR (1991) Cloud condensation nuclei near marine stratus. J Geophys Res 96:20795-20808

Lelieveld J, Heintzenberg J (1992) Sulphate cooling effect on climate through in-cloud oxidation of anthropogenic $\mathrm{SO}_{2}$. Science 258:117-120

Lohmann U, Feichter J (2005) Global indirect aerosol effects: a review. Atmos Chem Phys 5:715-737

Maps of India (2016) Drought prone areas in India, http://www. mapsofindia.com/maps/india/drought-prone-areas.html

Misra AK (2010) Modeling the depletion of dissolved oxygen in a lake due to submerged macrophytes. Nonlinear Anal Model Control 15(2):185-198

Misra AK, Verma M, Venturino E (2015) Modeling the control of atmospheric carbon dioxide through reforestation: effect of time delay. Model Earth Syst Environ 1:24 (17 pages)

Misra AK (2016) Effects of aerosols in making artificial rain: a modeling study. J Math Chem 54:1596-1611

Narasimhan R (2002) How is artificial rain produced. The Hindu, www.hinduonnet.com/thehindu/seta/2002/05/30/stories/2002053 000190300.htm

Pandis SN, Seinfeld JH, Pilinis C (1990) Chemical composition differences in fog and cloud droplets of different sizes. Atmos Environ 24A:1954-1969 
Pöschl U (2005) Atmospheric Aerosols: Composition, Transformation, Climate and Health Effects. Angew Chem Int Ed 44:7520-7540

Pruppacher HR, Klett JD (1997) Microphysics of clouds and precipitation, 2nd edn. Kluwer Acad Publications, Dordrecht

Saxena VK, Grovenstein JD (1994) The role of clouds in the enhancement of cloud condensation nuclei concentrations. Atmos Res 31:71-89

Shaw G (1989) Production of condensation nuclei in clean air by nucleation of $\mathrm{H}_{2} \mathrm{SO}_{4}$. Atmos Environ 23:2841-2846

Shukla JB, Sundar S, Misra AK, Naresh R (2008) Modelling the removal of gaseous pollutants and particulate matters from the atmosphere of a city by rain: effect of cloud density. Environ Model Assess 13:255-263
Shukla JB, Misra AK, Naresh R, Chandra P (2010) How artificial rain can be produced? A mathematical model. Nonlinear Anal RWA 11:2659-2668

Shukla JB, Sundar S, Misra AK, Naresh R (2013) Modeling the effects of aerosols to increase rainfall in regions with shortage. Meteorol Atmos Phys 120:157-163

Sundar S, Naresh R, Misra AK, Shukla JB (2009) A nonlinear mathematical model to study the interactions of hot gases with cloud droplets and raindrops. Appl Math Model 33:3015-3024

Sundar S, Sharma RK (2013) The role of aerosols to increase rainfall in the regions with less intensity rain: A modeling study. Comput Ecol Software 3(1):1-8

Xinhua News Agency(2006) China makes artificial rain for Beijing, www.chinadaily.com.cn/china/2006-05/06/content-583222.html 